\title{
A radial Phragmén-Lindelöf estimate for plurisubharmonic functions on algebraic varieties
}

\author{
by RüDiger W. Braun (Düsseldorf), Reinhold Meise (Düsseldorf) \\ and B. A. TAYlor (Ann Arbor, MI) \\ Dedicated to Professor Dr. H.-G. Tillmann \\ on the occasion of his 75th birthday
}

\begin{abstract}
For complex algebraic varieties $V$, the strong radial Phragmén-Lindelöf condition (SRPL) is defined. It means that a radial analogue of the classical PhragménLindelöf Theorem holds on $V$. Here we derive a sufficient condition for $V$ to satisfy (SRPL), which is formulated in terms of local hyperbolicity at infinite points of $V$. The latter condition as well as the extension of local hyperbolicity to varieties of arbitrary codimension are introduced here for the first time. The proof of the main result is based on a local version of the inequality of Sibony and Wong. The property (SRPL) provides a priori estimates which can be used to deduce more refined Phragmén-Lindelöf results for algebraic varieties.
\end{abstract}

1. Introduction. A pure dimensional algebraic variety $V$ in $\mathbb{C}^{n}$ is said to satisfy the strong radial Phragmén-Lindelöf condition (SRPL) if there are constants $A \geq 1, B \geq 0$ such that each plurisubharmonic function $u$ on $V$ which satisfies

( $\alpha) u(z) \leq|z|+o(|z|), z \in V$

( $\beta) u(x) \leq 0, x \in V \cap \mathbb{R}^{n}$,

also satisfies

$(\gamma) u(z) \leq A|z|+B, z \in V$.

If $V$ is homogeneous and if for each irreducible component $W$ of $V$ the real dimension of $W \cap \mathbb{R}^{n}$ equals the complex dimension of $V$, then the

1991 Mathematics Subject Classification: Primary 32F05, 31C10.

Key words and phrases: Sibony-Wong inequality, Phragmén-Lindelöf principle.

The third author gratefully acknowledges support of the A. v. Humboldt Stiftung for visits at Mathematisches Institut der Heinrich-Heine-Universität Düsseldorf, where part of this research was carried out. 
Phragmén-Lindelöf Theorem for the half-plane in $\mathbb{C}$ implies

$$
u(z) \leq|\operatorname{Im} z| \leq|z| \quad \text { for each } z \in \mathbb{C} \cdot\left(V \cap \mathbb{R}^{n}\right)
$$

whenever $u$ is plurisubharmonic on $V$ and satisfies $(\alpha)$ and $(\beta)$. Since $\mathbb{C} \cdot\left(V \cap \mathbb{R}^{n}\right)$ is nonpluripolar in each component of $V$, it follows from a theorem of Sibony and Wong [17] (see also Siciak [19] and Meise, Taylor, and Vogt [10], 3.1) that $u$ satisfies $(\gamma)$ for some $A \geq 1$ depending on $V$ and with $B=0$. Thus, irreducible homogeneous varieties satisfy (SRPL) if and only if the real points in the variety have "maximal dimension".

In the present paper we investigate what happens in the case of varieties which are not homogeneous. For example, when

$$
V=\left\{z \in \mathbb{C}^{3}: z_{1}^{5}+z_{2}^{5}+z_{3}^{5}=1\right\}
$$

and $u$ is plurisubharmonic on $V$, satisfying $(\alpha)$ and $(\beta)$, how can we find any global bound on $u$ ? In this example, there are a handful of complex lines in $V$, e.g., $\{(1,0,0)+\zeta(0,1,-1): \zeta \in \mathbb{C}\}$, but only very few. So the argument used for homogeneous varieties cannot be applied and we do not know whether it can be used at all. Nevertheless, the variety $V$ in the example satisfies (SRPL) as a consequence of the following general theorem.

1.1. TheOREM. Let $P_{m} \in \mathbb{C}\left[z_{1}, \ldots, z_{n}\right]$ be a homogeneous polynomial of degree $m$ with real coefficients and assume that $P_{m}$ has the following properties:

(a) each irreducible factor of $P_{m}$ has an $(n-1)$-dimensional set of real zeros,

(b) there are no repeated factors in the irreducible factorization of $P_{m}$.

Then for each polynomial $q \in \mathbb{R}\left[z_{1}, \ldots, z_{n}\right]$ of degree less than $m$, the variety $V=\left\{z \in \mathbb{C}^{n}:\left(P_{m}+q\right)(z)=0\right\}$ satisfies (SRPL).

This theorem can also be interpreted in terms of extremal functions similar to those investigated by Siciak [18], [19]. In this setting, our theorem states that the extremal function of $V \cap \mathbb{R}^{n}$ in the set of all plurisubharmonic functions satisfying $(\alpha)$ on $V$ grows at most like a linear function near infinity.

Theorem 1.1 extends some of the results of Bainbridge [2]. It is sharp in the following sense: If condition (a) is not satisfied then $V$ fails (SRPL) for $q=0$. If $P_{m}$ has repeated irreducible factors then there exists $q \in$ $\mathbb{R}\left[z_{1}, \ldots, z_{n}\right]$ so that $V$ fails (SRPL). In this case the problem of determining which perturbations preserve (SRPL) is still open. A more detailed discussion of examples and open problems is given in Section 6 .

The restriction to varieties of codimension 1 is not essential and was chosen only for a first exposition. In fact, our main result is a sufficient condition for algebraic varieties in $\mathbb{C}^{n}$ of pure dimension at least 1 . To formulate this 
condition, we extend the concept of local hyperbolicity - due to Anderssonto points at infinity and higher codimension. Instead of working in projective space, which would also be a natural choice, we embed $V$ into $\mathbb{C}^{n+1}$ as the complex cone over $\{1\} \times V$. The projective closure of $V$ then corresponds to $\bar{V}:=\overline{\mathbb{C}(\{1\} \times V)}$, and the infinite points of $V$ are of the form $\widehat{\xi}=(0, \xi)$. We say that $V$ is locally hyperbolic at an infinite point $\widehat{\xi} \in \bar{V} \cap \mathbb{R}^{n+1}$ if there are a neighborhood $U$ of $\widehat{\xi}$ in $\bar{V}$ and a projection $\pi: \mathbb{R}^{n+1} \rightarrow \mathbb{R}^{n+1}$ whose kernel is a subspace of $\{0\} \times \mathbb{R}^{n}$ with codim $\operatorname{ker} \pi=\operatorname{dim} \bar{V}$, such that the complexification $\pi_{\mathbb{C}}$ satisfies: $\operatorname{ker} \pi_{\mathbb{C}}$ is transversal to $\bar{V}$ in $\widehat{\xi}$ and whenever for $y \in \bar{V} \cap U$ the projection $\pi_{\mathbb{C}}(y)$ is real, then $y$ is already real. Our Main Theorem 5.1 states that $V$ satisfies (SRPL) whenever each irreducible component of $V_{\infty}$ contains at least one infinite point at which $V$ is locally hyperbolic. The latter condition, however, is not necessary, as an example of Bainbridge [2] shows (see 6.3).

In conclusion, we want to indicate that our motivation to investigate (SRPL) comes from the characterization problem for varieties $V$ that satisfy Phragmén-Lindelöf conditions, in particular the conditions $\operatorname{PL}\left(\mathbb{R}^{n}, \omega\right)$ and (SPL) which characterize the existence of continuous linear right inverses for linear partial differential operators (see Meise, Taylor, and Vogt [12], [11] and Palamodov [15]). It was used by Meise and Taylor [8] and in [4] and [5]. In all these applications, the property (SRPL) serves as an a priori estimate which is then refined using local considerations.

2. Preliminaries. In this section we introduce the radial PhragménLindelöf conditions (SRPL) and (RPL) and fix the notation for dealing with points at infinity.

2.1. Definition. Let $V$ be an algebraic variety of pure dimension $k$ in $\mathbb{C}^{n}$ and $\Omega$ be an open subset of $V$. A function $u: \Omega \rightarrow[-\infty, \infty[$ will be called plurisubharmonic if it is locally bounded above, plurisubharmonic in the usual sense on $\Omega_{\mathrm{reg}}$, the set of all regular points of $V$ in $\Omega$, and satisfies

$$
u(z)=\limsup _{\xi \in \Omega_{\mathrm{reg}}, \xi \rightarrow z} u(\xi)
$$

at the singular points of $V$ in $\Omega$. $\operatorname{By} \operatorname{PSH}(\Omega)$ we denote the set of all plurisubharmonic functions on $\Omega$.

Next we introduce the property (SRPL) of algebraic varieties which is studied in this paper.

2.2. Definition. Let $V$ be an algebraic variety of pure dimension $k \geq 1$ in $\mathbb{C}^{n}$. We say that $V$ satisfies $\operatorname{SRPL}(A, B)$ for $A \geq 1, B \geq 0$ if the following holds: Every $u \in \operatorname{PSH}(V)$ which satisfies $(\alpha)$ and $(\beta)$ also satisfies $(\gamma)$ :

$(\alpha) u(z) \leq|z|+o(|z|), z \in V,|z| \rightarrow \infty$, 
(及) $u(x) \leq 0, x \in V \cap \mathbb{R}^{n}$

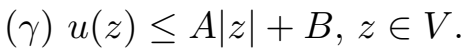

We say that $V$ satisfies (SRPL), the strong radial Phragmén-Lindelöf condition, if $\operatorname{SRPL}(A, B)$ holds on $V$ for some $A \geq 1, B \geq 0$.

2.3. Remark. The terminology (SRPL) comes from Meise, Taylor, and Vogt [10], where a weaker condition (RPL) was characterized. We recall that a pure dimensional algebraic variety $V$ in $\mathbb{C}^{n}$ satisfies the radial PhragménLindelöf condition (RPL) if there exists $A \geq 1$ such that for each $\varrho>0$ there exists $B_{\varrho}>0$ such that each $u \in \operatorname{PSH}(V)$ which satisfies $(\alpha)$ and $(\beta)$ also satisfies $(\gamma)$ :

( $\alpha) u(z) \leq|z|+o(|z|), z \in V$,

( $\beta) u(z) \leq \varrho|\operatorname{Im} z|, z \in V$,

$(\gamma) u(z) \leq A|z|+B_{\varrho}, z \in V$.

The main result of [10], Theorem 5.1, shows that a pure dimensional algebraic variety $V$ in $\mathbb{C}^{n}$ satisfies (RPL) if and only if its cone $V_{\mathrm{h}}$ of limiting directions at infinity has (RPL), which is equivalent to the fact that the real points in $V_{\mathrm{h}}$ have maximal dimension in the sense of Definition 2.5 below.

The cone of limiting directions at infinity $V_{\mathrm{h}}$ is of course nothing else than the set of infinite points of the projective closure of $V$. We will switch between both descriptions, analytic and algebraic, in the sequel. However, to avoid usage of coordinate charts for projective space $\mathbb{P}^{n}$, we will work with homogeneous varieties in $\mathbb{C}^{n+1}$ instead.

2.4. Points at infinity. Let $V$ be an algebraic variety in $\mathbb{C}^{n}$ of pure dimension $k \geq 1$. Its vanishing ideal is defined by

$$
I(V):=\left\{f \in \mathbb{C}\left[z_{1}, \ldots, z_{n}\right]: f(z)=0 \text { for all } z \in V\right\} .
$$

The homogenization of a polynomial $P \in \mathbb{C}\left[z_{1}, \ldots, z_{n}\right]$ of degree $m$ is defined by

$$
P_{\mathrm{h}}\left(z_{0}, \ldots, z_{n}\right):=z_{0}^{m} P\left(z_{1} / z_{0}, \ldots, z_{n} / z_{0}\right) .
$$

The elements of the form $P_{\mathrm{h}}$ with $P \in I(V)$ constitute the vanishing ideal of a variety $\bar{V}$ in $\mathbb{C}^{n+1}$, given by

$$
\bar{V}:=\left\{z=\left(z_{0}, \ldots, z_{n}\right) \in \mathbb{C}^{n+1}: P_{\mathrm{h}}(z)=0 \text { for all } P \in I(V)\right\} .
$$

The corresponding variety $\left\{\left(z_{0}: \ldots: z_{n}\right) \in \mathbb{P}^{n}: z \in \bar{V}, z \neq 0\right\}$ is the projective closure of $V$. For arbitrary $t \neq 0$ the slice $\left\{z \in \bar{V}: z_{0}=t\right\}$ is isomorphic to $V$. We define the set of infinite points of $V$ by

$$
V_{\infty}:=\left\{z \in \bar{V}: z_{0}=0\right\} .
$$


Another way to deal with infinity is via the cone of limiting directions, defined as

$$
V_{\mathrm{h}}:=\left\{r \lim _{j \rightarrow \infty} z_{j} /\left|z_{j}\right|: r \geq 0, z_{j} \in V,\left|z_{j}\right| \rightarrow \infty\right\} .
$$

Since the Zariski and euclidean closures of $\bar{V} \backslash V_{\infty}$ coincide (see Mumford [13], Theorem 2.33), we have

$$
V_{\infty}=\{0\} \times V_{\mathrm{h}}
$$

With each point $\zeta \in V_{\mathrm{h}}$ we associate the point $\widehat{\zeta}:=\left(0, \zeta_{1}, \ldots, \zeta_{n}\right) \in V_{\infty}$. For a polynomial $P$ of degree $m$ it is customary to denote the principal part by $P_{m}$. With this notation it is easy to give another description of $V_{\mathrm{h}}$ :

$$
V_{\mathrm{h}}=\left\{z \in \mathbb{C}^{n}: P_{\operatorname{deg} P}(z)=0 \text { for all } P \in I(V)\right\} \text {. }
$$

In order to derive necessary conditions for (SRPL) we recall the definition of the dimension condition which was already used in Hörmander [7] and Meise, Taylor, and Vogt [10], 2.6, [12], 3.11.

2.5. Definition. Let $V$ be a pure dimensional variety in $\mathbb{C}^{n}$. We say that $V$ satisfies the dimension condition if for every irreducible component $W$ of $V$, the real dimension of $W \cap \mathbb{R}^{n}$ is equal to the complex dimension of $V$.

Note that $V$ satisfies the dimension condition if and only if it is the smallest complex algebraic variety that contains $V \cap \mathbb{R}^{n}$.

2.6. Proposition. Let $V$ be a pure dimensional algebraic variety in $\mathbb{C}^{n}$.

(a) If $V$ is homogeneous then the following conditions are equivalent:

(i) $V$ satisfies (SRPL),

(ii) $V$ satisfies (RPL),

(iii) $V$ satisfies the dimension condition.

(b) If $V$ satisfies (SRPL) then $V_{\mathrm{h}}$ satisfies (SRPL).

Pr o of. (a) Obviously, (SRPL) implies (RPL). When $V$ is homogeneous, a standard scaling argument shows that $(\mathrm{RPL})$ is equivalent to $\operatorname{SRPL}(A, 0)$ for some $A \geq 1$. Hence the equivalence between (ii) and (iii) follows from Meise, Taylor, and Vogt [10], Theorem 5.1.

(b) Since (SRPL) implies (RPL), this follows from [10], Theorem 5.1, and (a).

REMARK. The necessary condition for $V$ to satisfy (SRPL) given by Proposition 2.6(b) is not sufficient. To see this, consider the zero variety $V$ of the polynomial $x^{2} y-1$. It satisfies (RPL) by Meise, Taylor, and Vogt [10], 5.1. Note that $u: V \rightarrow \mathbb{R}, u(x, y)=|\operatorname{Im} \sqrt{y}|$, is in $\operatorname{PSH}(V)$ and satisfies $u(z)=o(|z|)$ as well as $u(z)=0$ for $z \in V \cap \mathbb{R}^{2}$. Therefore, $\lambda u$ satisfies $2.2(\alpha)$ and $(\beta)$ for each $\lambda>0$. This implies that $V$ cannot satisfy (SRPL). 
In Theorem 5.1 we will give a condition which implies that the converse implication in Proposition 2.6(b) holds. To prove this, we will use the following proposition, which is an immediate consequence of the definition of plurisubharmonic functions on a variety.

2.7. Proposition. A pure dimensional algebraic variety $V$ in $\mathbb{C}^{n}$ satisfies (SRPL) if and only if each irreducible component $W$ of $V$ satisfies (SRPL).

3. A local Sibony-Wong inequality. In this section we prove that the theorem of Sibony-Wong [17] can be extended to certain plurisubharmonic functions that are only defined on suitable open subcones of a homogeneous algebraic variety. This extension will be the key step in the proof of the Main Theorem 5.1 given in Section 5 below.

For the formulation of the result we recall that a subset $K$ of $\mathbb{C}^{n}$ is a complex cone if $\lambda K \subset K$ for each $\lambda \in \mathbb{C}$. By abuse of notation, a cone $K$ is called open if the intersection with the unit sphere $S$ in $\mathbb{C}^{n}$ is open in $S$. We also recall that a subset $E$ of some open subset $\Omega$ of an algebraic variety $V$ is pluripolar in $\Omega$ if for at least one irreducible component $\Omega_{0}$ of $\Omega$, the set $E \cap \Omega_{0}$ is pluripolar in $\Omega_{0}$.

3.1. TheOREM. Let $W$ be a homogeneous algebraic variety of pure dimension $k$ in $\mathbb{C}^{n}$ and let $\pi: W \rightarrow \mathbb{C}^{k}, \pi\left(z^{\prime}, z^{\prime \prime}\right)=z^{\prime}$, be proper. Let $\Gamma^{\prime} \subset \mathbb{C}^{k}$ be an open complex cone, let $\Gamma:=\pi^{-1}\left(\Gamma^{\prime}\right)$, and let $R \subset \Gamma$ be a complex cone which is nonpluripolar in $\Gamma$. Then there exists a constant $A \geq 1$ such that for each $u \in \operatorname{PSH}(\Gamma)$ which is positively homogeneous and satisfies

(a) $u(z) \leq|z|$ for $z \in R$,

(b) $v\left(z^{\prime}\right):=\max \left\{u\left(z^{\prime}, z^{\prime \prime}\right):\left(z^{\prime}, z^{\prime \prime}\right) \in \Gamma\right\}$ for $z^{\prime} \in \Gamma^{\prime}$, extends to a plurisubharmonic function $\widetilde{v}$ on $\mathbb{C}^{k}$

each extension $\widetilde{v}$ satisfies

(c) $\widetilde{v}\left(z^{\prime}\right) \leq A\left|z^{\prime}\right|, z^{\prime} \in \mathbb{C}^{k}$.

In particular, $u$ satisfies $u(z) \leq A|z|$ for all $z \in W$.

Before proving Theorem 3.1, we recall the concept of an extremal function which will be used in the proof and also later on.

3.2. Definition. Let $W$ be a pure dimensional algebraic variety in $\mathbb{C}^{n}, \Omega$ an open subset of $W$, and $E \neq \emptyset$ a subset of $\Omega$. Then $U_{E}=U_{E}(\cdot, \Omega): \Omega \rightarrow \mathbb{R}$ is defined by

$$
U_{E}(z):=\sup \{u(z): u \in \operatorname{PSH}(\Omega), 0 \leq u \leq 1, u \leq 0 \text { on } E\} .
$$

The upper semicontinuous regularization $U_{E}^{*}$ of $U_{E}$, given by 


$$
U_{E}^{*}(z)=U_{E}^{*}(z, \Omega):=\lim \sup \left\{U_{E}(\zeta): \zeta \rightarrow z, \zeta \in \Omega\right\},
$$

is called the extremal plurisubharmonic function of $E$ relative to $\Omega$.

Proof of Theorem 3.1. Fix some complex cone $K$ in $\Gamma^{\prime}$ which is closed and has nonempty interior. Then let

$$
L:=\sup \left\{v\left(z^{\prime}\right): z^{\prime} \in K,\left|z^{\prime}\right| \leq 1\right\},
$$

and fix an extension $\widetilde{v}$ according to (b). Since $v$ is positively homogeneous on $\Gamma^{\prime}$, we have

$$
v\left(z^{\prime}\right) \leq L\left|z^{\prime}\right| \quad \text { for all } z^{\prime} \in K .
$$

Since $K$ is nonpluripolar in $\mathbb{C}^{k}$, Theorem 1 of Sibony-Wong [17], in the formulation of Siciak [19], Corollary 11.2, implies the existence of $\gamma=\gamma(K)$ such that

$$
\widetilde{v}\left(z^{\prime}\right) \leq L \gamma\left|z^{\prime}\right| \quad \text { for all } z^{\prime} \in \mathbb{C}^{k} .
$$

By the definition of $\widetilde{v}$, we get

$$
\sup \{u(z): z \in \Gamma,|\pi(z)| \leq 1\} \leq \sup \left\{\widetilde{v}\left(z^{\prime}\right):\left|z^{\prime}\right| \leq 1\right\} \leq L \gamma .
$$

Now let

$$
\Omega:=\{z \in \Gamma: 0<|\pi(z)|<2\}, \quad E:=\{z \in R \backslash\{0\}:|\pi(z)| \leq 1\},
$$

and note that there exists $M \geq 1$ such that

$$
|z| \leq M|\pi(z)| \quad \text { for all } z \in W .
$$

Since the complex cone $R$ is nonpluripolar in $\Gamma$, the set $E$ is nonpluripolar in $\Omega$. Hence, the extremal function $U_{E}(\cdot, \Omega)$ is nontrivial and therefore

$$
\mu:=\sup \left\{U_{E}^{*}(z, \Omega): \pi(z) \in K,|\pi(z)|=1\right\}<1 .
$$

Next note that $\log u$ is plurisubharmonic, since $u$ is plurisubharmonic and positively homogeneous (compare the proof of Meise, Taylor, and Vogt [10], 5.5), and that for $z \in \Omega$,

$$
\log u(z)=\log 2+\log u(z / 2) \leq \log 2+\log L \gamma .
$$

For $z \in E$ condition (a) implies

$$
\log u(z) \leq \log |z| \leq \log M+\log |\pi(z)| \leq \log M .
$$

From this and the definition of the extremal function we get

$$
\log u(z)-\log M \leq(\log 2+\log L \gamma) U_{E}^{*}(z), \quad z \in \Omega .
$$

Taking the supremum over $z \in \Gamma$ with $\pi(z) \in K,|\pi(z)| \leq 1$, it follows that

$$
\log L \leq(\log 2+\log L \gamma) \mu+\log M
$$

and consequently

$$
\log L \leq \frac{\mu(\log 2+\log \gamma)+\log M}{1-\mu}
$$


Hence $L$ does not depend on $v$. Therefore, the conclusion (c) follows from (3.1), while the second conclusion follows from the first one and the definition of $v$.

4. Local hyperbolicity at infinity. In this section, we extend the notion of local hyperbolicity in the sense of Andersson [1] (see also Hörmander [7], 6.4) to higher codimension as well as to points at infinity. This will be used to formulate the main result 5.1.

4.1. Localizations and tangent varieties. For a polynomial $P$ its localization $P_{x}$ at $x$ is defined as the lowest order homogeneous term of $\tau \mapsto P(x+\tau)$. For a variety $V$ in $\mathbb{C}^{n}$ the tangent cone at $x \in V$ is defined to be

$$
T_{x} V:=\left\{t \in \mathbb{C}^{n}: P_{x}(t)=0 \text { for all } P \in I(V)\right\} .
$$

This is the tangent cone $C_{3}(V, x)$ in Whitney's sense [21]. At some places, we will also use Whitney's tangent cone $C_{4}$. A vector $v \in \mathbb{C}^{n}$ is an element of $C_{4}(V, x)$ if and only if there are sequences of points $q_{i}$ regular in $V$ and vectors $v_{i}$ tangent to $V$ at $q_{i}$ such that $v=\lim _{i \rightarrow \infty} v_{i}$.

If $N$ is a subspace of $\mathbb{R}^{n}$, then we denote by $N_{\mathbb{C}}$ its complexification $N_{\mathbb{C}}=\operatorname{span}_{\mathbb{C}} N$. We say that $N_{\mathbb{C}}$ is transversal at $x$ to a family $\left(P_{\alpha}\right)_{\alpha \in A}$ of polynomials if the following holds: If for some $t \in N_{\mathbb{C}}$ we have $\left(P_{\alpha}\right)_{x}(t)=0$ for all localizations $\left(P_{\alpha}\right)_{x}, \alpha \in A$, then $t=0$.

4.2. Definition. Let $N$ be a subspace of $\mathbb{R}^{n}$ and fix $x \in \mathbb{R}^{n}$. A family $\left(P_{\alpha}\right)_{\alpha \in A}$ of polynomials in $\mathbb{C}\left[z_{1}, \ldots, z_{n}\right]$ is called locally hyperbolic at $x$ with respect to $N$ if the following two conditions hold:

(1) $N_{\mathbb{C}}$ is transversal to $\left(P_{\alpha}\right)_{\alpha \in A}$,

(2) there is a neighborhood $U$ of $x$ such that if $y \in U \cap\left(\mathbb{R}^{n}+N_{\mathbb{C}}\right)$ satisfies $P_{\alpha}(y)=0$ for all $\alpha \in A$, then $y \in \mathbb{R}^{n}$.

REMARK. In the case of a single polynomial, this definition coincides with Andersson's [1] as given by Hörmander [7], 6.4.

In the following definition we refer to the notation introduced in 2.4.

4.3. Definition. Let $V \subset \mathbb{C}^{n}$ denote an algebraic variety of pure dimension $k \geq 1$. It is said to be locally hyperbolic at $x \in V \cap \mathbb{R}^{n}$ with respect to an $(n-k)$-dimensional subspace $N$ of $\mathbb{R}^{n}$ if $I(V)$ is locally hyperbolic at $x$ with respect to $N$.

REMARK. It is easy to see that this is the case if and only if one system of generators $\left(P_{\alpha}\right)_{\alpha \in A}$ of the ideal $I(V)$ is locally hyperbolic at $x$. The same statement is true if "one system" is replaced by "all systems".

4.4. Definition. Let $V \subset \mathbb{C}^{n}$ denote an algebraic variety of pure dimension $k \geq 1$. Then $V$ is said to be locally hyperbolic at an infinite point 
$\widehat{\xi} \in V_{\infty} \cap \mathbb{R}^{n+1}$ if there is an $(n-k)$-dimensional subspace $N$ of $\{0\} \times \mathbb{R}^{n}$ such that $\left(P_{\mathrm{h}}\right)_{P \in I(V)}$ is locally hyperbolic at $\widehat{\xi}$ with respect to $N$.

REMARK. It is possible that there is no $(n-k)$-dimensional subspace of $\{0\} \times \mathbb{R}^{n}$ which is transversal to $\left(P_{\mathrm{h}}\right)_{P \in I(V)}$ at $x$. By definition, $V$ is not locally hyperbolic at an infinite point $\widehat{\xi}$ then. The next two results show that this is a reasonable definition. Proposition 4.6 is also needed for the proof of the Main Theorem 5.1.

4.5. Lemma. Let $W \subset \mathbb{C}^{n}$ be an analytic subset of a neighborhood of 0 , let $\pi: \mathbb{C}^{n} \rightarrow \mathbb{C}^{k}$ be defined by $\pi(z):=\left(z_{1}, \ldots, z_{k}\right)$ and assume that $\pi: W \rightarrow$ $\widetilde{W}$ is a branched cover. Assume, furthermore, that $T_{0} W \cap \operatorname{ker} \pi \neq\{0\}$. Then, for every neighborhood $U$ of 0 , there is $z \in W \cap U \backslash \mathbb{R}^{n}$ with $\pi(z)$ real.

Proof. Assume the conclusion is false. Then there is a neighborhood $U$ of 0 such that if for $z \in W \cap U$ the projection $\pi(z)$ is real, then $z$ is real. Assume that $U$ is a polycylinder such that $\left.\pi\right|_{W \cap U}$ is still a branched covering and define a plurisubharmonic function $\phi$ on $\pi(U) \subset \mathbb{C}^{k}$ by

$$
\phi(\zeta):=\max \{|\operatorname{Im} z|: z \in W \cap U, \pi(z)=\zeta\} .
$$

Note that $\phi$ is bounded since $\pi$ is proper and that $\phi(\zeta)=0$ whenever $\zeta$ is real. Classical estimates for the harmonic measure of the half-disk (see Nevanlinna [14], 38) imply the existence of $A>0$ such that

$$
\phi(\zeta) \leq A|\operatorname{Im} \zeta| \quad \text { for } \zeta \text { near } 0 .
$$

Fix $(0, v) \in T_{0} W \cap \operatorname{ker} \pi$ with $|\operatorname{Im} v|=1$. By Chirka [6], §8.5, Proposition 2, there is a $C^{1}$-curve $\alpha$ in $W$ with $\alpha(0)=0$ and $\dot{\alpha}(0)=v$. Write $\alpha(t)=\left(\alpha_{1}(t), \alpha_{2}(t)\right)$ with $\alpha_{1}(t) \in \mathbb{C}^{k}$ and $\alpha_{2}(t) \in \mathbb{C}^{n-k}$. Then $\pi \circ \alpha=\alpha_{1}$, $\dot{\alpha}_{1}(0)=0$, and $\dot{\alpha}_{2}(0)=v$. In particular, $\left|\alpha_{1}(t)\right|=o(t)$ and $\left|\alpha_{2}(t)-t v\right|=o(t)$. Thus $\phi\left(\alpha_{1}(t)\right) \geq|\operatorname{Im} \alpha(t)| \geq t-o(t)$, while $\left|\operatorname{Im} \alpha_{1}(t)\right| \leq o(t)$. This contra$\operatorname{dicts}(4.1)$.

At a given point $\widehat{\xi} \in V_{\infty}$, there may be a complicated singularity of $\bar{V}$. However, arbitrarily close to $\widehat{\xi}$ there is a point $\widehat{\zeta} \in V_{\infty}$ where the structure of $\bar{V}$ can easily be described, as Stutz [20] has pointed out. We are going to quote Stutz's work from Chirka [6]. The following proposition says that, for almost all $\widehat{\xi} \in V_{\infty} \cap \mathbb{R}^{n+1}$, local hyperbolicity means that $\bar{V}$ is a union of graphs near $\widehat{\xi}$.

4.6. Proposition. Let $V \subset \mathbb{C}^{n}$ be an algebraic variety of pure dimension $k \geq 1$ and let $\xi \in \mathbb{R}^{n}, \xi \neq 0$, be a regular point of $V_{\mathrm{h}}$ with $\operatorname{dim} C_{4}(\bar{V}, \widehat{\xi})=k+1$. Assume that there is a neighborhood $U$ of $\hat{\xi}$ such that $U \cap \operatorname{sing} \bar{V}=U \cap V_{\infty}$. Let $\pi: \mathbb{C}^{n} \rightarrow \mathbb{C}^{n}$ be a projection whose kernel and range are both spanned by real vectors, and define $\widetilde{\pi}: \mathbb{C}^{n+1} \rightarrow \mathbb{C}^{n+1}$ 
by $\widetilde{\pi}\left(x_{0}, x^{\prime}\right)=\left(x_{0}, \pi\left(x^{\prime}\right)\right)$. Assume that $\left.\widetilde{\pi}\right|_{U \cap \bar{V}}$ is a branched cover and that $z \in U \cap \bar{V}$ is real whenever $\tilde{\pi}(z)$ is real.

Then there are $\varepsilon>0$, a neighborhood $U_{1} \subset \mathbb{C}^{n}$ of $\xi$, and a biholomorphic map $F: U_{1} \rightarrow F\left(U_{1}\right)$ satisfying $F\left(U_{1} \cap \mathbb{R}^{n}\right)=F\left(U_{1}\right) \cap \mathbb{R}^{n}$. Also, for $\eta:=$ $F(\xi)$, there are a neighborhood $U_{2}$ of $\left(0, \eta_{1}, \ldots, \eta_{k}\right), l \in \mathbb{N}$, and holomorphic functions $f_{1}, \ldots, f_{l}: U_{2} \rightarrow \mathbb{C}^{n-k}$ with $f_{j}\left(U_{2} \cap \mathbb{R}^{k+1}\right) \subset \mathbb{R}^{n-k}$ such that

$$
\begin{aligned}
& \left\{x=\left(x_{0}, x^{\prime}\right):\left|x_{0}\right|<\varepsilon, x^{\prime} \in F\left(\bar{V} \cap U_{1}\right)\right\} \\
& \quad=\left\{\left(x_{0}, \ldots, x_{k}, f_{j, 1}(x), \ldots, f_{j, n-k}(x)\right): x \in U_{2}, 1 \leq j \leq l\right\} .
\end{aligned}
$$

In particular, $V$ is locally hyperbolic in $\widehat{\xi}$ with respect to a Zariski open and dense set of $(n-k)$-dimensional subspaces of $\{0\} \times \mathbb{R}^{n}$, where the topology is that of the Grassmannian.

Proof. Let $W$ be a representative of an irreducible component of the germ $[\bar{V}]_{\widehat{\xi}}$. We claim first that $W$ is regular at $\widehat{\xi}$.

Assume for contradiction that $W$ is singular at $\widehat{\xi}$. We have $\operatorname{dim} C_{4}(W, \widehat{\xi})$ $=k+1$ since $C_{4}(W, \widehat{\xi}) \subset C_{4}(\bar{V}, \widehat{\xi})$ and the dimension is at least $k+1$. By hypothesis, $W$ is irreducible at $\widehat{\xi}$, hence it follows from $\operatorname{dim} C_{4}(W, \widehat{\xi})=k+1$, by Chirka [6], $\S 9.5$, Proposition 2, that the dimension of $\operatorname{sing} W$ at $\widehat{\xi}$ is $k$. Since $\operatorname{sing} \bar{V}$ is near $\widehat{\xi}$ an irreducible analytic set of dimension $k$ containing $\operatorname{sing} W$, we deduce, after possibly shrinking $U$, that $U \cap \operatorname{sing} W=U \cap \operatorname{sing} \bar{V}$.

We may assume that the projection is $\pi: z \mapsto\left(z_{1}, \ldots, z_{k}, 0, \ldots, 0\right)$. By Lemma 4.5, no tangent to $\bar{V}$ at $\widehat{\xi}$ lies in ker $\widetilde{\pi}$ since otherwise there would be a contradiction to the hypothesis. Since $V_{\infty}$ is regular at $\widehat{\xi}$, this means that we can assume

(4.3) $V_{\infty} \cap U_{3}=\left\{\left(0, z_{1}, \ldots, z_{k}, g_{1}(z), \ldots, g_{n-k}(z)\right): z\right.$ close to $\left.\left(\xi_{1}, \ldots, \xi_{k}\right)\right\}$, and it is clear from the hypotheses that the $g_{j}$ are real-valued for real arguments. So after a change of coordinates of the form described in the claim,

$$
(\operatorname{sing} W) \cap U=V_{\infty} \cap U=\left(\widehat{\xi}+\left(\{0\} \times \mathbb{C}^{k} \times\{0\}^{n-k}\right)\right) \cap U .
$$

It is shown in Chirka [6], $\S 9.5$, Corollary 1 , that $C_{4}(W, \widehat{\xi})=T_{\widehat{\xi}} W$ and that it is a $(k+1)$-dimensional plane. From this and Lemma 4.5 it follows that

$$
C_{4}(W, \widehat{\xi}) \cap \operatorname{ker} \widetilde{\pi}=T_{\widehat{\xi}} W \cap \operatorname{ker} \tilde{\pi}=\{0\} .
$$

By (4.4), the hypothesis implies

$$
((\operatorname{sing} W)-\widehat{\xi}) \cap \operatorname{ker} \tilde{\pi}=\{0\} .
$$

We have arranged coordinates so that $\operatorname{ker} \widetilde{\pi}=\{0\}^{k+1} \times \mathbb{C}^{n-k}$. Now we have verified the hypotheses of Stutz's Theorem [20], 4.2 (see [6], § 9.5, Proposition 1). Hence there are a neighborhood $U_{4}$ of $\widehat{\xi}, \kappa \in \mathbb{N}, r>0$, and 
holomorphic functions $\phi_{1}, \ldots, \phi_{n-k}$ such that

$$
\begin{aligned}
& W \cap U_{4} \\
= & \left\{\widehat{\xi}+\left(r \tau_{0}^{\kappa}, r \tau_{1}, \ldots, r \tau_{k}, \tau_{0}^{\kappa} \phi_{1}(\tau), \ldots, \tau_{0}^{\kappa} \phi_{n-k}(\tau)\right): \tau \in \mathbb{C}^{k+1} \text { small }\right\} .
\end{aligned}
$$

We may assume that $\kappa$ is the smallest positive integer for which (4.5) holds. By assumption, $\kappa \geq 2$. Since $\kappa$ is minimal, there must be $\tau_{1}, \ldots, \tau_{k} \in$ $\mathbb{R}$ arbitrarily small and $j \in\{1, \ldots, n-k\}$ such that the Taylor series expansion of $\tau_{0} \mapsto \phi_{j}\left(\tau_{0}, \tau_{1}, \ldots, \tau_{k}\right)$ contains a power $\tau_{0}^{q}$ where $q$ is not a multiple of $\kappa$. We choose $\tau_{0}$ to be a small positive constant times a primitive root of unity if $\kappa>2$ and small and purely imaginary if $\kappa=2$. In both cases, this yields a contradiction to the hypothesis that $z$ is real if $\widetilde{\pi}(z)$ is real.

This shows the claim that $W$ is regular in $\widehat{\xi}$. We may again assume that the projection is $\pi: z \mapsto\left(z_{1}, \ldots, z_{k}, 0, \ldots, 0\right)$. Then Lemma 4.5 , applied in $n+1$ variables, shows that $W$ is actually a graph of the form claimed in (4.2).

In earlier drafts of the present paper and in Bainbridge [2], the notion of hyperbolicity at infinity in a direction was used instead of local hyperbolicity at an infinite point. This concept is also useful for the proof of the Main Theorem 5.1. To show that both concepts are equivalent we start with a remark.

4.7. Regular coordinate systems. Let $V$ be a pure dimensional algebraic variety of dimension $k$ in $\mathbb{C}^{n}$. Coordinates $z=(w, s) \in \mathbb{C}^{k} \times \mathbb{C}^{n-k}$ are called regular for $V$ if there exists $C>0$ such that $|s| \leq C(1+|w|)$ for all $(w, s) \in V$.

4.8. Lemma. If $\pi: \mathbb{C}^{k} \times \mathbb{C}^{n-k} \rightarrow \mathbb{C}^{k},(w, s) \mapsto w$, denotes regular coordinates for $V$, then

$$
\widetilde{\pi}: \bar{V} \rightarrow \mathbb{C}^{k+1}, \quad x \mapsto\left(x_{0}, \ldots, x_{k}\right),
$$

is proper.

Pro of. Note that the regularity of the coordinate system implies

$$
|y| \leq C(1+|\pi(y)|)+|\pi(y)| \quad \text { for all } y \in V .
$$

Let $x=\left(x_{0}, \ldots, x_{k}\right) \in \mathbb{C}^{k+1}$ be given. Assume first $x_{0} \neq 0$ and fix $y=$ $\left(y_{0}, y^{\prime}\right) \in \bar{V}$ with $\widetilde{\pi}(y)=x$. Then $y^{\prime} / y_{0} \in V$, in particular

$$
\left|\frac{y^{\prime}}{y_{0}}\right| \leq C\left(1+\left|\pi\left(\frac{y^{\prime}}{y_{0}}\right)\right|\right)+\left|\pi\left(\frac{y^{\prime}}{y_{0}}\right)\right|
$$

and hence $\left|y^{\prime}\right| \leq(C+1)|x|$. Now assume $x_{0}=0$. Then there are $r>0$ and $y^{(j)} \in V, j \in \mathbb{N}$, with $\left|y^{(j)}\right| \rightarrow \infty$ and

$$
y^{\prime}=r \lim _{j \rightarrow \infty} \frac{y^{(j)}}{\left|y^{(j)}\right|} .
$$


Note that $x=\pi(y)=r \lim _{j \rightarrow \infty} \pi\left(y^{(j)}\right) /\left|y^{(j)}\right|$. Hence (4.7) implies

$$
\left|y^{\prime}\right|=|r| \leq \limsup _{j \rightarrow \infty} \frac{\left|y^{(j)}\right|}{\left|\pi\left(y^{(j)}\right)\right|} \leq C+1 .
$$

4.9. Definition. Let $V$ be an algebraic variety of pure dimension $k \geq 1$, and fix $\xi \in V_{\mathrm{h}} \cap \mathbb{R}^{n}$ with $|\xi|=1$. Then $V$ is hyperbolic at infinity in direction $\xi$ if there is a choice of regular coordinates $z=(w, s)$ for $V$ and constants $R>1$, $\delta>0$ such that, in the complex cone $\Gamma(\xi, \delta)$ about the origin, given by

$$
\Gamma(\xi, \delta)=\left\{z \in \mathbb{C}^{n}:|z-\langle z, \xi\rangle \xi|<\delta|\langle z, \xi\rangle|\right\},
$$

if $z=(w, s) \in V \cap \Gamma(\xi, \delta),|w|>R$, and $w$ is real, then $s$ is also real.

4.10. Corollary. Assume that the hypotheses of Proposition 4.6 hold for $\widehat{\xi}$ with $|\xi|=1$. Then $V$ is locally hyperbolic at infinity in direction $\xi$ for every coordinate system such that for $\pi: z=(w, s) \mapsto w$ we have $T_{\widehat{\xi}} \bar{V} \cap \operatorname{ker} \widetilde{\pi}=\{0\}$.

Proof. The cones with truncated tips $\Gamma(\xi, \delta) \backslash B_{R}(0), \delta, R>0$, constitute a neighborhood basis of $\widehat{\xi}$ in the following sense: For every neighborhood $U$ of $\widehat{\xi}$ in $\mathbb{C}^{n+1}$ there are $\delta, R>0$ such that

$$
\left\{z=\left(z_{0}, z^{\prime}\right) \in \mathbb{C}^{n+1}: z^{\prime} \in \Gamma(\xi, \delta), 0<\left|z_{0}\right|<\delta,\left|z^{\prime}\right|>R\left|z_{0}\right|\right\} \subset U,
$$

and for each choice of $\delta, R>0$ there is a neighborhood $U$ of $\widehat{\xi}$ in $\mathbb{C}^{n+1}$ such that

$$
\begin{aligned}
& U \backslash\left(\{0\} \times \mathbb{C}^{n}\right) \\
& \quad \subset\left\{z=\left(z_{0}, z^{\prime}\right) \in \mathbb{C}^{n+1}: z^{\prime} \in \Gamma(\xi, \delta), 0<\left|z_{0}\right|<\delta,\left|z^{\prime}\right|>R\left|z_{0}\right|\right\} .
\end{aligned}
$$

From this, the claim is immediate.

4.11. Proposition. Let $V$ be an algebraic variety of pure dimension $k \geq 1$, and let $W$ be an irreducible component of $V_{\mathrm{h}}$. The following are equivalent:

(1) there is $\widehat{\xi} \in(\{0\} \times W) \cap \mathbb{R}^{n+1}$ such that $V$ is locally hyperbolic in $\widehat{\xi}$,

(2) there is $\xi \in W \cap \mathbb{R}^{n}$ with $|\xi|=1$ such that $V$ is hyperbolic at infinity in direction $\xi$.

Proof. Let $W_{\infty}:=\{0\} \times W$. We first show that (1) implies (2). If $N$ is as in the definition of local hyperbolicity, any projection $\pi$ with $\operatorname{ker} \pi=N_{\mathbb{C}}$ is proper in a neighborhood of $\widehat{\xi}$. All further neighborhoods will be subsets of this one. It follows directly from the definition that the real dimension of $W_{\infty} \cap \mathbb{R}^{n+1}$ at $\widehat{\xi}$ is $k$. Thus there is $\widehat{\zeta} \in W_{\infty} \cap \mathbb{R}^{n+1}$ arbitrarily close to $\widehat{\xi}$ such that $V_{\infty}$ is regular at $\widehat{\zeta}$.

CASE 1: $\widehat{\zeta} \in \operatorname{sing} \bar{V}$ and the dimension of $W_{\infty} \cap \operatorname{sing} \bar{V}$ in $\widehat{\zeta}$ is $k$. Both $\operatorname{sing} \bar{V}$ and $V_{\infty}$ are regular of dimension $k$ in $\widehat{\zeta}$, in particular, their germs are 
irreducible. Since their intersection also has dimension $k$, both germs must coincide. Hence there is a neighborhood $U_{1}$ of $\widehat{\zeta}$ such that $U_{1} \cap \operatorname{sing} \bar{V}=$ $U_{1} \cap V_{\infty}$. There is $\widehat{\eta} \in W_{\infty} \cap U_{1} \cap \mathbb{R}^{n+1}$ such that $V_{\mathrm{h}}$ is regular in $\eta$ and $\operatorname{dim} C_{4}(\bar{V}, \widehat{\eta})=k+1$, since by [6], Lemma 9.4, the set of $\widehat{\eta}$ where $\operatorname{dim} C_{4}(\bar{V}, \widehat{\eta})>k+1$ has dimension at most $k-1$. Now the claim follows from Corollary 4.10.

CAsE 2: $\widehat{\zeta} \notin \operatorname{sing} \bar{V}$ or the dimension of $W_{\infty} \cap \operatorname{sing} \bar{V}$ in $\widehat{\zeta}$ is strictly less than $k$. Then there is $\eta \in W \cap \mathbb{R}^{n}$ such that $\widehat{\eta}$ is a regular point of $\bar{V}$. The claim follows from Lemma 4.5.

The proof of the converse is the same except that Proposition 4.6 is used instead of Corollary 4.10. However, the part of the hypothesis of Proposition 4.6 dealing with inverse images of real points is not a priori satisfied at infinite points. Thus, we have to show first that the variety $V_{\mathrm{h}}$ is locally hyperbolic in $\xi$. To do so, assume that $\pi(\zeta)=\left(\zeta_{1}, \ldots, \zeta_{k}\right)$ is the projection given by the regular coordinates from Definition 4.9 and define $\widetilde{\pi}\left(z_{0}, z^{\prime}\right)=\left(z_{0}, \pi\left(z^{\prime}\right)\right)$. Fix $\zeta \in V_{\mathrm{h}} \cap \Gamma(\xi, \delta / 2) \backslash B_{2 R}(0)$ such that $\pi(\zeta)$ is real. We have to show that $\zeta$ is real.

For small $t \in \mathbb{R}$, let $\alpha(t)=(t, \pi(\zeta))$ and choose a lift $\widetilde{\beta}(t)=(t, \beta(t)) \in \bar{V}$ with $\beta(0)=\zeta$ and $\pi \circ \beta=\alpha$. Then, for small $t, \widetilde{\beta}(t)$ is close to $\widehat{\zeta}$ and thus, as in the proof of $4.10, \beta(t)$ lies in $\Gamma(\xi, \delta)$. The hypothesis implies $\beta(t)$ real for $t \neq 0$ and hence, by continuity, $\beta(0)$ is real.

\section{The Main Theorem}

5.1. MAIN TheOREM. Let $V$ be an algebraic variety in $\mathbb{C}^{n}$ of pure dimension $k \geq 1$ which satisfies the following condition:

(1) For each irreducible component $W$ of $V_{\mathrm{h}}$ there exists $\xi \in W \cap \mathbb{R}^{n}$ such that $V$ is locally hyperbolic at infinity at $\widehat{\xi}$.

Then $V$ satisfies (SRPL).

REMARK. The condition of local hyperbolicity at infinity is not necessary in order that $V$ satisfies (SRPL). This follows from the example of Bainbridge [2], already mentioned in Section 1, which has (SRPL), but is not locally hyperbolic at any real infinite point (see Example 6.3).

Proof (of Theorem 5.1). Because of Proposition 2.7 we can assume that $V$ is irreducible. Let $W_{1}, \ldots, W_{q}$ denote the irreducible components of $V_{\mathrm{h}}$, so that

$$
V_{\mathrm{h}}=\bigcup_{j=1}^{q} W_{j} .
$$

By hypothesis, there exist $\xi_{j} \in W_{j} \cap\left(\mathbb{R}^{n} \backslash\{0\}\right)$ so that $V$ is locally hyperbolic 
at the infinite points $\widehat{\xi}_{j}$ for $1 \leq j \leq q$. In Proposition 4.11 it is shown that there are points in $W_{j}$, which we will again call $\xi_{j}$, such that $V$ is locally hyperbolic at infinity in direction $\xi_{j}$. By Corollary 4.10, there exists a common choice of regular coordinates for $V$ and of constants $\delta>0, R_{1} \geq 1$ such that for the corresponding projection map $\pi: V \rightarrow \mathbb{C}^{k}$ and each $z \in V \cap \Gamma\left(\xi_{j}, \delta\right)$ satisfying $|\pi(z)|>R_{1}, z$ is real whenever $\pi(z)$ is real.

Let $D \in \mathbb{C}\left[w_{1}, \ldots, w_{k}\right]$, homogeneous of degree $d$, be chosen so that $V_{\mathrm{h}}$ is unbranched over $\left\{w \in \mathbb{C}^{k}: D(w) \neq 0\right\}$. (See, e.g., Whitney [21], Chapter I, Corollary 8E, or Shafarevich [16], Chapter II, 6.2, Theorem 4.) For $0<\eta<1$ let

$$
S(\eta):=\left\{z=(w, s) \in \mathbb{C}^{n}:|D(w)| \leq \eta|w|^{d}\right\} .
$$

Choosing $\eta>0$ small enough, possibly shrinking $\delta>0$ and perturbing $\xi_{j}$ by choosing a different center for a smaller cone, and increasing $R_{1}$, we can assume that

$$
\bigcup_{j=1}^{q} \Gamma\left(\xi_{j}, \delta\right) \subset V_{\mathrm{h}} \backslash S(\eta)
$$

and that the properties mentioned above still hold.

Since $V_{\mathrm{h}}$ is unbranched over $\pi(S(\eta))$,

$$
c(\eta)=\inf _{j \neq l}\left\{\left|\beta_{j}(w)-\beta_{l}(w)\right|:|w|=1, w \notin \pi(S(\eta))\right\}>0 .
$$

By the homogeneity of the $\beta_{j}$, this implies

$$
\min _{j \neq l}\left|\beta_{j}(w)-\beta_{l}(w)\right| \geq c(\eta)|w|, \quad w \notin \pi(S(\eta)) .
$$

Thus, outside $S(\eta)$ the $\beta_{j}(w)$ are distinct and at least $c(\eta)|w|$ apart, while on the other hand it is easy to see that for each $\alpha_{j}(w)$ there exists some $\beta_{k}(w)$ such that $\left|\alpha_{j}(w)-\beta_{k}(w)\right| \leq C(1+|w|)^{1-\varepsilon}$. Therefore, if $R(\eta)>1$ is chosen so that $C(1+|w|)^{1-\varepsilon}<\frac{1}{2} c(\eta)|w|$ for all $w \in \mathbb{C}^{k} \backslash S(\eta)$ satisfying $|w| \geq R(\eta)$, then there exists a unique $\beta_{i}(w)$ such that

$$
\left|\alpha_{j}(w)-\beta_{i}(w)\right|=\min _{1 \leq l \leq m^{\prime}}\left|\alpha_{j}(w)-\beta_{l}(w)\right| .
$$

Consequently, outside the exceptional set

$$
S^{*}(\eta):=\left\{z=(w, s) \in \mathbb{C}^{n}:|D(w)| \leq \eta|w|^{d} \text { or }|w| \leq R(\eta)\right\}
$$

the projection map $\pi$ induces an analytic map

$$
\tilde{\pi}: V \backslash S^{*}(\eta) \rightarrow V_{\mathrm{h}} \backslash S^{*}(\eta) .
$$

Note that for $\eta>0$ small enough, the sets $W_{j} \backslash S(\eta), 1 \leq j \leq q$, are connected. Hence

$$
\begin{aligned}
R(j):=\mathbb{C} \cdot\left(\Gamma\left(\xi_{j}, \delta\right) \cap \mathbb{R}^{n} \cap W_{j}\right) \text { is nonpluripolar } \\
\\
\quad \text { in } W_{j} \backslash S(\eta), 1 \leq j \leq q .
\end{aligned}
$$


Let

$$
\mathcal{R}:=\bigcup_{j=1}^{q} R(j)
$$

fix $\eta>0$ so small that (5.1) and (5.4) hold and choose $R_{0} \geq \max \left(R_{1}, R_{1}(\eta)\right)$ so large that the set

$$
\Omega:=\left\{z \in V:|\pi(z)|<2 R_{0}\right\}
$$

is connected.

To prove that the variety $V$ has the property (SRPL), let $u \in \operatorname{PSH}(V)$ be any function that satisfies $2.2(\alpha)$ and $(\beta)$. To show that $u$ satisfies $2.2(\gamma)$ for some constants $A \geq 1, B \geq 0$ which do not depend on $u$, let

$$
M(u):=\max \left\{u(z): z \in V,|\pi(z)| \leq R_{0}\right\} .
$$

The argument has two steps. First we show that there exists $A \geq 1$ depending only on $V, V_{\mathrm{h}}$, and $\eta$ so that

$$
u(z) \leq A|\pi(z)|+M(u), \quad z \in V .
$$

Then we show that there exists $B \geq 0$, not depending on $u$, so that $M(u)$ $\leq B$. This and (5.6) obviously imply that $u$ satisfies condition $2.2(\gamma)$, hence $V$ has $\operatorname{SRPL}(A, B)$.

To prove (5.6) for a suitable constant $A \geq 1$ we define the functions $u_{0}$ on $V_{\mathrm{h}} \backslash S^{*}(\eta)$ and $\widetilde{u}$ on $V_{\mathrm{h}} \backslash S(\eta)$ by

$$
u_{0}(z):=\max \{u(\zeta): \zeta \in V, \widetilde{\pi}(\zeta)=z\}, \quad \widetilde{u}(z):=\max \left\{u_{0}(z)-M(u), 0\right\} .
$$

Then $u_{0}$ is plurisubharmonic on $V_{\mathrm{h}} \backslash S^{*}(\eta)$ since $\widetilde{\pi}$ is analytic, while $\widetilde{u}$ is plurisubharmonic because of the definition of $M(u)$. Also define $v_{0}$ and $\widetilde{v}$ on $\mathbb{C}^{k}$ by

$$
v_{0}(w):=\max \{u(z): z \in V, \pi(z)=w\}, \quad \widetilde{v}(w):=\max \left\{v_{0}(w)-M(u), 0\right\} .
$$

By Hörmander [7], 4.4, $v_{0}$ and hence $\widetilde{v}$ are plurisubharmonic on $\mathbb{C}^{k}$. Note that $\widetilde{v}$ is a plurisubharmonic extension of

$$
w \mapsto \max \left\{\widetilde{u}(z): z \in V_{\mathrm{h}} \backslash S(\eta), \pi(z)=w\right\} .
$$

Next recall that $u$ satisfies

$$
u(z) \leq|z|+o(|z|) \text { and } u(x) \leq 0, \quad x \in V \cap \mathbb{R}^{n} .
$$

From the definition of $\tilde{\pi}$ in (5.3) we know that for $z \in V_{\mathrm{h}} \backslash S^{*}(\eta)$ and each $\zeta \in V \backslash S^{*}(\eta)$ satisfying $\tilde{\pi}(\zeta)=z$, we have

$$
|\widetilde{\pi}(\zeta)-z| \leq C(1+|w|)^{1-\varepsilon} .
$$

Hence (5.7), the regularity of the coordinate system, and the definition of $u_{0}$ imply

$$
\widetilde{u}(z) \leq|z|+o(|z|), \quad z \in V_{\mathrm{h}} \backslash S(\eta) .
$$


If $x \in \Gamma\left(\xi_{j}, \delta\right) \cap \mathbb{R}^{n} \cap W_{j}$ for some $j$, then $\widetilde{u}(x) \leq 0$ because $V$ is locally hyperbolic at infinity in the direction $\xi_{j}$. From this, (5.8) and the PhragménLindelöf theorem for subharmonic functions on $\mathbb{C}$, it follows that

$$
\widetilde{u}(z) \leq|\operatorname{Im} z| \leq|z| \quad \text { for all } z \in \mathcal{R} .
$$

Next define $\phi: V_{\mathrm{h}} \backslash S(\eta) \rightarrow[-\infty, \infty[$ by

$$
\phi(z)=\sup _{\zeta \in \mathbb{C}} \frac{\widetilde{u}(\zeta z)}{|\zeta|}, \quad z \in V_{\mathrm{h}} \backslash S(\eta) .
$$

Then $\phi$ is positive homogeneous. Since $\widetilde{u}$ is plurisubharmonic, the upper regularization $\phi^{*}$ of $\phi$ is plurisubharmonic and positive homogeneous. For $z \in \mathcal{R}$ we have $\phi(z) \leq|z|$ because of (5.9). Hence (5.4) implies

$$
\phi^{*}(z) \leq|z| \quad \text { for } z \in \mathcal{R} .
$$

Now define similarly to $\phi$ the function $\psi: \mathbb{C}^{k} \rightarrow[-\infty, \infty[$ by

$$
\psi(w)=\sup _{\zeta \in \mathbb{C}} \frac{\widetilde{v}(\zeta w)}{|\zeta|}, \quad w \in \mathbb{C}^{k} .
$$

Then $\psi^{*}$ is positive homogeneous and plurisubharmonic on $\mathbb{C}^{k}$. Moreover, for $w \in \mathbb{C}^{k} \backslash \pi(S(\eta))$ and $\zeta \in \mathbb{C}$, the definitions of $\widetilde{v}$ and $\widetilde{u}$ imply

$$
\frac{1}{|\zeta|} \widetilde{v}(\zeta w)=\frac{1}{|\zeta|} \max \left\{\widetilde{u}(\zeta z): z \in V_{\mathrm{h}} \backslash S(\eta), \pi(z)=w\right\} .
$$

Hence $\psi$ is an extension of $w \mapsto \max \left\{\phi(z): z \in V_{\mathrm{h}} \backslash S(\eta), \pi(z)=w\right\}$ and consequently $\psi^{*}$ extends $w \mapsto \max \left\{\phi^{*}(z): z \in V_{\mathrm{h}} \backslash S(\eta), \pi(z)=w\right\}$. Therefore we can now apply Theorem 3.1 with $W=V_{\mathrm{h}}, \Gamma=V_{\mathrm{h}} \backslash S(\eta)$, $\Gamma^{\prime}=\mathbb{C}^{k} \backslash \pi(S(\eta)), u=\phi^{*}$, and $v=\psi^{*}$. It gives the existence of $A \geq 1$ such that

$$
\psi^{*}(w) \leq A|w| \quad \text { for all } w \in \mathbb{C}^{k} .
$$

By the definition of $\psi^{*}$, this implies, for each $z \in V$,

$$
\left.u(z)-M(u) \leq \widetilde{v}(\pi(z)) \leq \psi(\pi(z)) \leq \psi^{*}(\pi(z)) \leq A \mid \pi(z)\right) \mid,
$$

which proves (5.6).

To prove that $M(u)$ is bounded above by some $B \geq 0$ not depending on $u$, let

$$
E:=V \cap \mathbb{R}^{n} \cap \Omega .
$$

Then the hypothesis on $V$ implies that $E$ is nonpluripolar in the open set $\Omega$ defined above. Therefore, the extremal function $U_{E}^{*}(\cdot, \Omega)$ of $E$ relative to $\Omega$ is not constant. Hence

$$
\mu:=\max \left\{U_{E}^{*}(z, \Omega): z \in V,|\pi(z)| \leq R_{0}\right\}<1 .
$$

Next note that by (5.6),

$$
u(z) \leq A 2 R_{0}+M(u), \quad z \in \Omega,
$$


while (5.7) implies $u(z)=0$ for $z \in E$. Hence the definition of $U_{E}^{*}(\cdot, \Omega)$ implies

$$
u(z) \leq\left(2 A R_{0}+M(u)\right) U_{E}^{*}(z, \Omega), \quad z \in \Omega,
$$

and consequently $M(u) \leq\left(2 A R_{0}+M(u)\right) \mu$. This implies

$$
M(u) \leq \frac{2 A R_{0} \mu}{1-\mu} .
$$

Hence $V$ satisfies $\operatorname{SRPL}(A, B)$ for $B=2 A R_{0} \mu /(1-\mu)$.

We conclude this section by showing that Theorem 1.1 follows from the Main Theorem 5.1.

Proof of Theorem 1.1. Let $q=\sum_{j=0}^{m-1} q_{j}$ be the decomposition of $q$ into homogeneous polynomials so that $\operatorname{deg} q_{j}=j$ unless $q_{j}=0$. Then

$$
\bar{V}=\left\{z=\left(z_{0}, z^{\prime}\right) \in \mathbb{C}^{n+1}: P_{\mathrm{h}}(z)=0\right\},
$$

where

$$
P_{\mathrm{h}}\left(z_{0}, z^{\prime}\right)=P_{m}\left(z^{\prime}\right)+\sum_{j=0}^{m-1} z_{0}^{m-j} q_{j}\left(z^{\prime}\right) .
$$

If we write $P_{m}=\prod_{l=1}^{\lambda} Q_{l}$ where the $Q_{j} \in \mathbb{C}\left[z_{1}, \ldots, z_{n}\right]$ are irreducible and pairwise nonproportional, then the varieties $V\left(Q_{l}\right)$ are the components of $V_{\mathrm{h}}$. By hypothesis there is, for each $l$, a $\xi_{l} \in \mathbb{R}^{n}$ with $Q_{l}\left(\xi_{l}\right)=0$ and $\nabla Q_{l}\left(\xi_{l}\right) \neq 0$. Then

$$
\nabla P_{\mathrm{h}}\left(0, \xi_{l}\right)=\nabla P_{m}\left(\xi_{l}\right)=\left(\prod_{\nu \neq l} Q_{\nu}\left(\xi_{l}\right)\right) \nabla Q_{l}\left(\xi_{l}\right) \neq 0 .
$$

So some derivative, say $\partial P_{\mathrm{h}} / \partial z_{n}$, does not vanish at $\left(0, \xi_{l}\right)$. By the implicit function theorem there are a neighborhood $U$ of 0 and a holomorphic function $f$ with real Taylor coefficients such that

$$
\bar{V} \cap U=\left\{z \in U: z_{n}=f\left(z_{0}, \ldots, z_{n-1}\right)\right\} .
$$

This shows that $V$ is locally hyperbolic at the infinite point $\widehat{\xi}_{l}$ with respect to $N=\{0\}^{n} \times \mathbb{R}$.

Remark. From the proof of Theorem 5.1, one sees that the constant $A$ in the (SRPL) estimate $u(z) \leq A|z|+B$ depends on the size of the cones $\Gamma(\xi, \delta)$ in which $V$ is locally hyperbolic. However, in the situation of Theorem 1.1, $V=\left\{z \in \mathbb{C}^{n}:\left(P_{m}+q\right)(z)=0\right\}$, it is not difficult to show that the cones can be taken very large, in fact equal to $\left\{z \in \mathbb{C}^{n}: P_{m}(z)=0\right\} \backslash S(\eta)$. Therefore, the constant $A$ depends only on the principal part $P_{m}$ of $P_{m}+q$. A similar result holds for general varieties $V$ provided that the sheet numbers $m$ and $m^{\prime}$ for $\pi: V \rightarrow \mathbb{C}^{k}$ and $\pi: V_{\mathrm{h}} \rightarrow \mathbb{C}^{k}$ are the same, i.e., the map $\tilde{\pi}: V \rightarrow V_{\mathrm{h}}$ defined in (5.3) is biholomorphic outside a small exceptional set. We omit 
the proof, since we do not have current applications for the result, and since the proof is similar to the codimension 1 case.

The constant $B$ in the (SRPL) estimate necessarily depends on the perturbation of $V$ away from $V_{\mathrm{h}}$. In particular, in Theorem 1.1(b), if $K$ is a compact subset of the space of polynomials with real coefficients and degree less than $m$, there is a constant $B(K)$ such that $\operatorname{SRPL}(A, B(K))$ holds for the polynomials $P_{m}+q, q \in K$. However, the choice of $B$ made in (5.11) does not show this result without further work because of the lack of a good estimate for the constant $\mu<1$. To prove it, one needs to relate the local extremal functions $V_{E}^{*}(z, \Omega)$ with the global extremal function on $V_{\mathrm{h}}$ of the cones $R_{i}$ in $V_{\mathrm{h}}$. We omit the details, which are straightforward but lengthy.

6. Discussion of examples, open questions. The Main Theorem 5.1 states that a pure dimensional algebraic variety $V$ inherits (SRPL) from its cone $V_{\mathrm{h}}$ of limiting directions in $V$ at infinity provided in sufficiently many real limiting directions nearby inverse images of real points under suitable projections are all real. Therefore it is natural to ask what happens when some of the branches $\left(\alpha_{j}(w), w\right)$ in $V$ that cluster near real branches $\left(\beta_{k}(w), w\right)$ of $V_{\mathrm{h}}$ are real while some are complex. In this case, the property of vanishing at the real points of $V$ is not preserved when a function $u$ on $V$ is pushed forward to $V_{\mathrm{h}}$ under the map $\widetilde{\pi}$ in (5.3). A simple example of this situation is the zero variety $V$ of the polynomial

$$
P(x, y, s)=s^{3}(s-y)+x^{3} .
$$

In this case

$$
V_{\mathrm{h}}=\left\{(x, y, s) \in \mathbb{C}^{3}: s=0 \text { or } s=y\right\} .
$$

Hence $V_{\mathrm{h}}$ is the union of two hyperplanes in $\mathbb{C}^{3}$. Note that the coordinates $(x, y, s)=(w, s) \in \mathbb{C}^{2} \times \mathbb{C}$ are regular for $V$. Generically there are four zeros $s=\alpha_{j}(x, y)$ of $P$, one of which is near $s=y$, while three of them are near $s=0$, in the sense that they grow like a fractional power less than 1 in $|(x, y)|$. When $x$ and $y$ are both real and large, only one of the three roots of $P(x, y, s)=0$ near $s=0$ is real while two are complex. By [3], Theorem 1, the following holds:

\subsection{EXAmple. The variety}

$$
V=\left\{(x, y, s) \in \mathbb{C}^{3}: s^{3}(s-y)+x^{3}=0\right\}
$$

does not satisfy (SRPL).

This shows that the conclusion of the Main Theorem 5.1 can fail if $V$ is not locally hyperbolic at infinity for sufficiently many real infinite points. The example also shows that Theorem 1.1 can fail if $P_{m}$ has repeated irreducible factors. In this situation, however, more can be shown. 
6.2. ExAmple. Let $P_{m} \in \mathbb{C}\left[z_{1}, \ldots, z_{n}\right]$ be homogeneous of degree $m$ with real coefficients and assume that $V\left(P_{m}\right)$ satisfies the dimension condition. If $P_{m}$ has repeated irreducible factors then there exists $q \in \mathbb{R}\left[z_{1}, \ldots, z_{n}\right]$ of degree less than $m$ such that $V\left(P_{m}+q\right)$ fails (SRPL).

To prove this, assume that $P_{m}=\prod_{j=1}^{s} Q_{j}^{r_{j}}$, where $Q_{1}, \ldots, Q_{s}$ are the irreducible factors of $P_{m}$ and where $r_{1} \geq \ldots \geq r_{s}$ with $r_{1} \geq 2$. Since $V\left(P_{m}\right)$ satisfies the dimension condition, each polynomial $Q_{j}$ is real up to a complex factor. Therefore we can assume without restriction that the polynomials $Q_{1}, \ldots, Q_{s}$ have real coefficients. Then define $q:=\prod_{j=2}^{s} Q_{j}^{r_{j}}$ and note that

$$
P+q=\left(Q_{1}^{r_{1}}+1\right) \prod_{j=2}^{s} Q_{j}^{r_{j}} .
$$

By Proposition 2.7, $V(P+q)$ fails (SRPL) if $V\left(Q_{1}^{r_{1}}+1\right)$ fails (SRPL). To prove the latter, define $u_{\nu}: \mathbb{C}^{n} \rightarrow \mathbb{R}$ by $u_{\nu}(z):=\nu\left|\operatorname{Im} Q_{1}(z)\right|$. Then $u_{\nu}$ is plurisubharmonic and $u_{\nu}(x)=0$ for $x \in \mathbb{R}^{n}$, since $Q_{1}$ has real coefficients. For $z \in V\left(Q_{1}^{r_{1}}+1\right)$ we have $\left|Q_{1}^{r_{1}}(z)\right|=1$ and hence

$$
u_{\nu}(z)=\nu\left|\operatorname{Im} Q_{1}(z)\right| \leq \nu\left|Q_{1}(z)\right| \leq \nu .
$$

Hence $u_{\nu}$ satisfies the conditions $2.2(\alpha)$ and $(\beta)$ of (SRPL). If we assume that $V\left(Q_{1}^{r_{1}}+1\right)$ satisfies (SRPL) then we get the existence of $A \geq 1, B \geq 0$ such that

$$
u_{\nu}(z) \leq A|z|+B \quad \text { for all } z \in V\left(Q_{1}^{r_{1}}+1\right), \quad \nu \in \mathbb{N} .
$$

Since the equation $\lambda^{r_{1}}+1=0$ has nonreal solutions for each $r_{1} \geq 2$, we can find $\zeta \in V\left(Q_{1}^{r_{1}}+1\right)$ such that $\left|\operatorname{Im} Q_{1}(\zeta)\right|>0$. From this and (6.1) we get

$$
\nu\left|\operatorname{Im} Q_{1}(\zeta)\right| \leq A|\zeta|+B \quad \text { for all } \nu \in \mathbb{N} .
$$

This contradiction shows that $V\left(Q_{1}^{r_{1}}+1\right)$ fails (SRPL).

In view of Examples 6.1 and 6.2 and of Theorems 5.1 and 1.1 it is natural to pose the following problem.

Problem. Let $V$ be a pure dimensional algebraic variety in $\mathbb{C}^{n}$ for which $V_{\mathrm{h}}$ satisfies (RPL). Which conditions on $V$ and $V_{\mathrm{h}}$ characterize when $V$ inherits (SRPL) from $V_{\mathrm{h}}$ ?

To indicate that the solution of this problem might be rather subtle and cannot be obtained only by using the methods that were applied to prove the Main Theorem 5.1, we recall the following example of Bainbridge [2].

6.3. ExAmple. Let $P(x, y, s):=\left(s^{2}-x^{2}\right)^{2}-y\left(y^{2}-x^{2}\right)$. Then $V(P)$ satisfies (SRPL).

Note that in this example

$$
V_{\mathrm{h}}=\left\{(s, x, y) \in \mathbb{C}^{3}: s=x \text { or } s=-x\right\}
$$


is again the union of two hyperplanes in $\mathbb{C}^{3}$ and that again $(s, x, y)=(s, w)$ are regular coordinates for $V(P)$. However, now generically two of the four roots of $P$ cluster near the two different branches of $V_{\mathrm{h}}$. As Bainbridge [2] has shown, $V(P)$ is not locally hyperbolic in the sense of 4.9 in any direction. By Proposition 4.11 it is also not locally hyperbolic at any infinite point in the sense of 4.4. This can also be easily seen from Proposition 4.6 since at no regular point of $\bar{V}$ does $\bar{V}$ have the form given in (4.2).

\section{References}

[1] K. G. Andersson, Propagation of analyticity of solutions of partial differential equations with constant coefficients, Ark. Mat. 8 (1971), 277-302.

[2] D. Bainbridge, Phragmén-Lindelöf estimates for plurisubharmonic functions of linear growth, thesis, Ann Arbor, 1998.

[3] R. W. Braun, R. Meise and B. A. Taylor, An example concerning radial Phragmén-Lindelöf estimates for plurisubharmonic functions on algebraic varieties, Linear Topol. Spaces Complex Anal. 3 (1997), 24-29.

[4] - - - - , A perturbation result for linear differential operators admitting a global right inverse on $\mathcal{D}^{\prime}$, Pacific J. Math., to appear.

[5] -, - - - Algebraic varieties on which the classical Phragmén-Lindelöf estimates hold for plurisubharmonic functions, Math. Z., to appear.

[6] E. M. Chirka, Complex Analytic Sets, Kluwer, Dordrecht, 1989.

[7] L. Hörmander, On the existence of real analytic solutions of partial differential equations with constant coefficients, Invent. Math. 21 (1973), 151-183.

[8] R. Meise and B. A. Taylor, Phragmén-Lindelöf conditions for graph varieties, Results Math. 36 (1999), 121-148.

[9] R. Meise, B. A. Taylor and D. Vogt, Characterization of the linear partial operators with constant coefficients that admit a continuous linear right inverse, Ann. Inst. Fourier (Grenoble) 40 (1990), 619-655.

[10] - - - - Extremal plurisubharmonic functions of linear growth on algebraic varieties, Math. Z. 219 (1995), 515-537.

[11] - - - - Continuous linear right inverses for partial differential operators on nonquasianalytic classes and on ultradistributions, Math. Nachr. 180 (1996), 213-242.

[12] - - - - Phragmén-Lindelöf principles on algebraic varieties, J. Amer. Math. Soc. 11 (1998), 1-39.

[13] D. Mumford, Algebraic Geometry I, Complex Projective Varieties, Grundlehren Math. Wiss. 221, Springer, Berlin, 1976.

[14] R. Nevanlinna, Eindeutige analytische Funktionen, Springer, 1974.

[15] V. P. Palamodov, A criterion for splitness of differential complexes with constant coefficients, in: Geometrical and Algebraical Aspects in Several Complex Variables, C. A. Berenstein and D. C. Struppa (eds.), EditEL, 1991, 265-291.

[16] I. R. Shafarevich, Basic Algebraic Geometry 1, Springer, 1994.

[17] N. Sibony and P. Wong, Some results on global analytic sets, in: Séminaire Lelong-Skoda (Analyse), Lecture Notes in Math. 822, Springer, 1978-79, 221-237. 
[18] J. Siciak, Extremal plurisubharmonic functions in $\mathbb{C}^{n}$, Ann. Polon. Math. 39 (1981), 175-211.

[19] - Extremal Plurisubharmonic Functions and Capacities in $\mathbb{C}^{n}$, Sophia Kokyuroku in Mathematics 14, Tokyo, 1982.

[20] J. Stutz, Analytic sets as branched coverings, Trans. Amer. Math. Soc. 166 (1972), 241-259.

[21] H. Whitney, Complex Analytic Varieties, Addison-Wesley, 1972.

Mathematisches Institut Heinrich-Heine-Universität

Universitätsstraße 1

40225 Düsseldorf, Germany

E-mail: braun@cs.uni-duesseldorf.de meise@cs.uni-duesseldorf.de
Department of Mathematics University of Michigan Ann Arbor, MI 48109, U.S.A. E-mail: taylor@umich.edu

Reçu par la Rédaction le 19.11.1998

Révisé le 6.5.1999 